\title{
Human papillomavirus infection and its association with cervical dysplasia in Ecuadorian women attending a private cancer screening clinic
}

\author{
C.R. Brown ${ }^{1}$, M.L. Leon ${ }^{4}$, K. Muñoz ${ }^{4}$ A. Fagioni ${ }^{\dagger} \dagger$, L.G. Amador ${ }^{4}$, B. Frain ${ }^{3}$, W. Tu${ }^{1}$, \\ B. Qadadri ${ }^{1}$ and D.R. Brown ${ }^{1,2}$ \\ ${ }^{1}$ Department of Medicine, ${ }^{2}$ Department of Microbiology and Immunology, ${ }^{3}$ Department of Pathology, \\ Indiana University School of Medicine, Indianapolis, IN, USA \\ ${ }^{4}$ Society for the Fight against Cancer (SOLCA), Santa Elena, Guayas, Ecuador
}

Correspondence to: D.R. Brown, 545 Barnhill Drive, Indiana University School of Medicine, Indianapolis, IN 46202, USA

E-mail: darbrow@iupui.edu

\begin{abstract}
Women living in Latin American countries bear a disproportionate burden of cervical cancer, a condition caused by infection with the human papillomavirus (HPV). We performed a study in Santa Elena, Guayas (currently Santa Elena Province), Ecuador, to determine how often HPV could be detected in women attending a private cancer screening clinic. Participants underwent a Pap test, and vaginal and cervical swabs were performed for HPV testing by the polymerase chain reaction (PCR). Each participant completed a verbally administered survey. The mean age of 302 participants was 37.7 years (range 18 to 78 years). The majority of cervical and vaginal specimens contained sufficient DNA to perform PCR. Overall, $24.2 \%$ of the participants had either a cervical or vaginal swab that tested positive for HPV. In general, there was a good correlation between the HPV types detected in the cervical and vaginal swabs from the participants, but vaginal swabs were more likely to contain HPV DNA than were cervical swabs. The high-risk HPV types 16, 52, 58, and 59 and the low-risk HPV types 62, 71, 72, and 83 were the most frequently detected HPV types. The number of lifetime sexual partners was positively associated with detection of any HPV type, detection of oncogenic HPV, and abnormal Pap smears. Further studies are needed to determine if these results are representative of all Ecuadorian women and to determine if cervical cancers in Ecuadorian women are caused by the same HPV types found in the swab specimens obtained in this study.
\end{abstract}

Key words: Human papillomavirus; Cervical dysplasia; Ecuador

†In memoriam

Received November 5, 2008. Accepted May 4, 2009

\section{Introduction}

Human papillomavirus (HPV), the causative agent of cervical cancer, is the most common sexually transmitted infection. In addition to cervical cancer, HPV-related malignancies include vulvar cancer (1), vaginal cancer and anal cancer (2). Furthermore, approximately $45 \%$ of cases of penile cancer are associated with HPV (3). Of the approximately 100 different types of HPVs that have been identi- fied and fully sequenced, approximately 40 infect the genital tract $(4,5)$. The most common type of HPV associated with non-cervical genital tract cancers is HPV 16, which causes more than $50 \%$ of these cancers $(3,6)$.

The genital HPV types are divided into two categories, 'high risk' and 'low risk', originally assigned based on whether the HPV type could or could not be found as a solitary isolate in cervical cancer specimens. Individuals infected with highrisk HPV infection have an increased risk of cervical cancer. 
High-risk types such as HPV types 16, 18 and related HPV types cause dysplastic lesions of the cervix (manifested by abnormal Pap smears), which may progress to invasive cancer of the cervix in a subset of infected women (7). Women infected with only low-risk HPV types have a low risk of developing cervical cancer. The low-risk types such as HPV 6 and HPV 11 are associated with benign, proliferative lesions known as condylomata acuminata, and commonly referred to as genital warts (8-10). Because HPV is the causative agent of cervical cancer, knowledge of the epidemiology of HPV is critical.

Cervical cancer is caused by HPV infection, with approximately 500,000 overall cases of cervical cancer and about 275,000 deaths worldwide every year (11-14). Women in Latin American countries bear a disproportionate burden of cervical cancer (15). Rates of cervical cancer are approximately 30 per 100,000 in Ecuador, Venezuela, and Mexico in women aged 35 through 64 years, and rates are higher than 20 per 100,000 in several other Latin American countries (16). These rates far exceed those in the United States and Canada (approximately 4 per 100,000), and cervical cancer mortality in Latin American women is at least 4-fold higher than in the United States $(15,17)$.

Few studies have examined the epidemiology of HPV in Ecuador, a country in which routine Pap smears are generally not done, and a country with a high cervical cancer death rate $(15,18,19)$. We therefore performed a study in Santa Elena, Guayas, Ecuador, to determine how often HPV could be detected in women attending a private cancer screening clinic. We compared HPV detection in vaginal swabs and cervical swabs, and determined the types of HPV that were detected in these women. We also examined the association of abnormal Pap smear results with HPV detection.

\section{Material and Methods}

\section{Study population}

Female patients older than 18 years presenting to the Society for the Fight Against Cancer (SOLCA for its abbreviation in Spanish), a private cancer screening clinic in Santa Elena, Guayas, Ecuador, were asked to participate in the study. Approval of the Institutional Review Board at Indiana University School of Medicine was obtained for the study, as well as approval of officials of SOLCA. To be included, a subject had to be at least 18 years of age with an intact uterus, have a history of sexual intercourse with a male partner, and be able to provide informed consent. Subjects were enrolled between September 2005 and January 2006. Subjects with clinical evidence of gross cervicitis were excluded, as were individuals who were menstruating at the time of their visit. Written consent was obtained from each subject prior to her enrollment in the study. A copy of the signed consent form was given to the participant.

\section{Survey}

Each participant was asked to complete a short survey that was verbally administered. Specific questions were asked regarding age, marital status, the number of lifetime sexual partners, pregnancies, previous Pap smears, previous diagnosis of genital warts, and cigarette smoking.

\section{Procedures}

All participants underwent routine clinical procedures including history and physical exam. A Pap test, vaginal swab, and a cervical swab were obtained from all participants. The Pap test was performed by local clinicians using an Ayres spatula and a cytobrush to assess for abnormalities in the exfoliated cervical cells, and was interpreted at the SOLCA clinic. Participants with abnormal cytology results were treated according to the SOLCA clinic standard of care. Pap smears were also examined at a later time by a cytology technician at the Indiana University School of Medicine; no patient identification was provided to Indiana University. Examination of Pap smears at Indiana University was done solely for educational purposes, and had no bearing on patient care. No specific quality control of locally interpreted Pap smears was done.

For the endo/ectocervical specimen, a Pap smear was performed using an Ayers spatula, then the vaginal speculum was left in place. A DACRON ${ }^{\mathrm{TM}}$ swab was introduced into the cervical os with enough pressure to maintain contact with the epithelium but not to induce bleeding. The swab was twirled one to two times, and then back and forth across the ectocervix. The swab was then placed in the collection tube, the end of the shaft protruding from the tube was broken off, and the tube was sealed with the cap. The capped plastic tube was labeled with a number only and stored at $-20^{\circ} \mathrm{C}$ until ready for shipment. For the vaginal specimen, the DACRON ${ }^{\mathrm{TM}}$ swab was introduced into the vaginal vault, and moved back and forth in a zigzag motion along both lateral walls. The swab was then placed into the collection tube, and processed as for the cervical swab. The cervical/vaginal specimens were sent by $\mathrm{DHL}$ to the Indiana University School of Medicine.

\section{HPV detection}

The Roche polymerase chain reaction (PCR)/reverse blot strip assay was used for HPV typing (20). This assay uses a set of oligonucleotide primer pools PGMY09 and PGMY11 based on the same primer binding regions used 
for the MY09/11 degenerate primer PCR assay. Reactions were amplified in a Perkin Elmer TC9600 Thermal Cycler (Perkin Elmer Corp., USA) as previously described (21). Known positive and negative (no DNA) specimens were included in each assay as controls. To determine specimen adequacy, the GH20/PC04 human $\beta$-globin gene target was co-amplified with HPV sequences. The HPV immobilized probe array used to determine HPV type contains probe lines to detect individual HPV types and human $\beta$-globin control probes. High-risk HPV types detected in the assay are $16,18,26,31,33,35,39,45,51,52$, $53,56,58,59,66,67,68,70,73,82$, and IS39 (a partially characterized type). Low-risk types detected in the assay are $6,11,40,42,54,55,61,62,64,71,72,81,83,84$, and CP (a partially characterized type). Hybridization and detection were performed as described by the manufacturer (Roche Molecular Diagnostics, USA).

\section{Data analysis}

Subject characteristics, including age, marital status, the number of lifetime sexual partners, pregnancies, previous Pap smears, previous diagnosis of genital warts, and cigarette smoking were summarized. Relative frequencies of HPV infections, including both high-risk and low-risk types, were described graphically by a bar chart. Typespecific HPV detections in cervical and vaginal specimens were presented graphically. HPV test results were then cross-tabulated by sample collection methods (cervical and vaginal swabs) and Pap smear results [Normal, lowgrade squamous intraepithelial lesions (LGSIL), and highgrade squamous intraepithelial lesions (HGSIL)]. Agespecific prevalence rates of high-risk and any HPV infections were also reported. Logistic regression analyses were performed to identify correlates of high-risk HPV, any HPV infection, and abnormal Pap smear.

\section{Results}

\section{Demographics and clinical characteristics of study participants}

A total of 311 women completed the survey and participated in the study, including Pap smear testing and specimen collection. Overall, we included 302 (97.1\%) of all participants in the analysis for HPV, as these participants had both cervical and vaginal specimens that were positive for $\beta$-globin, and therefore had adequate specimens for HPV analysis, and had a Pap smear performed. No participant experienced an adverse event as a result of the study.

The mean age of these 302 participants was 37.7 years (range 18 to 78 years). For marital status, 263 (88.3\%) were married or in a common law union, 35 (11.7\%) were single or widowed, and four participants gave no response to this question. The mean age $( \pm S D$ ) of first sexual intercourse was $18.1 \pm 3.8$ years.

The mean number of lifetime sex partners was $1.7 \pm$ 1.4 ; 181 participants $(61.4 \%)$ reported 1 lifetime sex partner, $73(24.8 \%)$ reported 2 lifetime sex partners, 21 (7.1\%) reported 3 lifetime sex partners, 5 participants $(1.7 \%)$ reported 4 lifetime partners, and 15 participants (5.0\%) reported 5 or more lifetime sex partners. Seven participants did not answer this question.

The mean number of pregnancies for the participants was $4.3 \pm 3.1$, with a range of zero to 15 pregnancies. Pap smears had been previously performed on 235 , or $77.8 \%$ of study participants. A history of an abnormal Pap smear was reported by 17 (5.6\%) participants. A history of genital warts was reported by $26(8.6 \%)$ participants. Nineteen participants $(6.3 \%)$ smoked cigarettes.

HPV detection in all study participants

Of all specimens collected, $99.0 \%$ of the cervical and $98.0 \%$ of the vaginal specimens contained sufficient DNA to perform PCR, based on amplification of the human $\beta$-globin gene. As indicated above, a total of 302 participants had adequate specimens from both the cervix and vagina, and had Pap smears performed. Women among these 302 participants were considered to be positive for HPV if either the cervical or vaginal swab was positive for HPV. Overall, 73 women, or $24.2 \%$ of all participants tested positive for HPV (Table 1). High-risk HPV types were detected in 44 women (14.6\%). The most frequently detected high-risk HPV types were HPV 16, 52, 58, and 59 (Figure 1).

Low-risk HPV types were detected in one or both swab specimens from 44 women (14.6\%). The most frequently detected low-risk HPV types were HPV types 62, 71, 72, and 83 (Figure 1). Both high- and low-risk HPV types were detected in 15 women (5.0\%). A mean of 1.4 different HPV types was detected in each HPV-positive woman.

\section{HPV detection according to type of swab}

In addition to considering rates of HPV detection in the study participants, we also considered the frequency of HPV detection in vaginal and cervical swabs (Table 1). This was done to determine if vaginal swabs (which women can perform without supervision from a doctor or nurse) and cervical swabs (which must be done by a clinician) would provide the same or similar HPV type distribution.

Of the cervical swabs from the 302 participants, 43 , or $14.2 \%$ were positive for any HPV (Table 1 ). Thirty-one cervical swabs, or $10.3 \%$, were positive for 1 or more highrisk HPV types, and 15 cervical swabs, or $5.0 \%$ were positive for 1 or more low-risk HPV types. Of 302 vaginal 
Table 1. Detection of human papillomavirus (HPV) in cervical and vaginal swabs and in participants.

\begin{tabular}{llllr}
\hline & Any HPV & HR-HPV & LR-HPV & HR-HPV + LR-HPV \\
\hline Cervical swabs $(\mathrm{N}=302)$ & $43(14.2 \%)$ & $31(10.3 \%)$ & $15(5.0 \%)$ & $3(1.0 \%)$ \\
Vaginal swabs $(\mathrm{N}=302)$ & $55(18.2 \%)$ & $36(11.9 \%)$ & $43(14.2 \%)$ & $12(4.0 \%)$ \\
Participants $(\mathrm{N}=302)$ & $73(24.2 \%)$ & $44(14.6 \%)$ & $44(14.6 \%)$ & $15(5.0 \%)$ \\
\hline
\end{tabular}

Data are reported as number with percent in parentheses. HR-HPV = high-risk or oncogenic HPV; LR-HPV = low-risk or nononcogenic HPV.

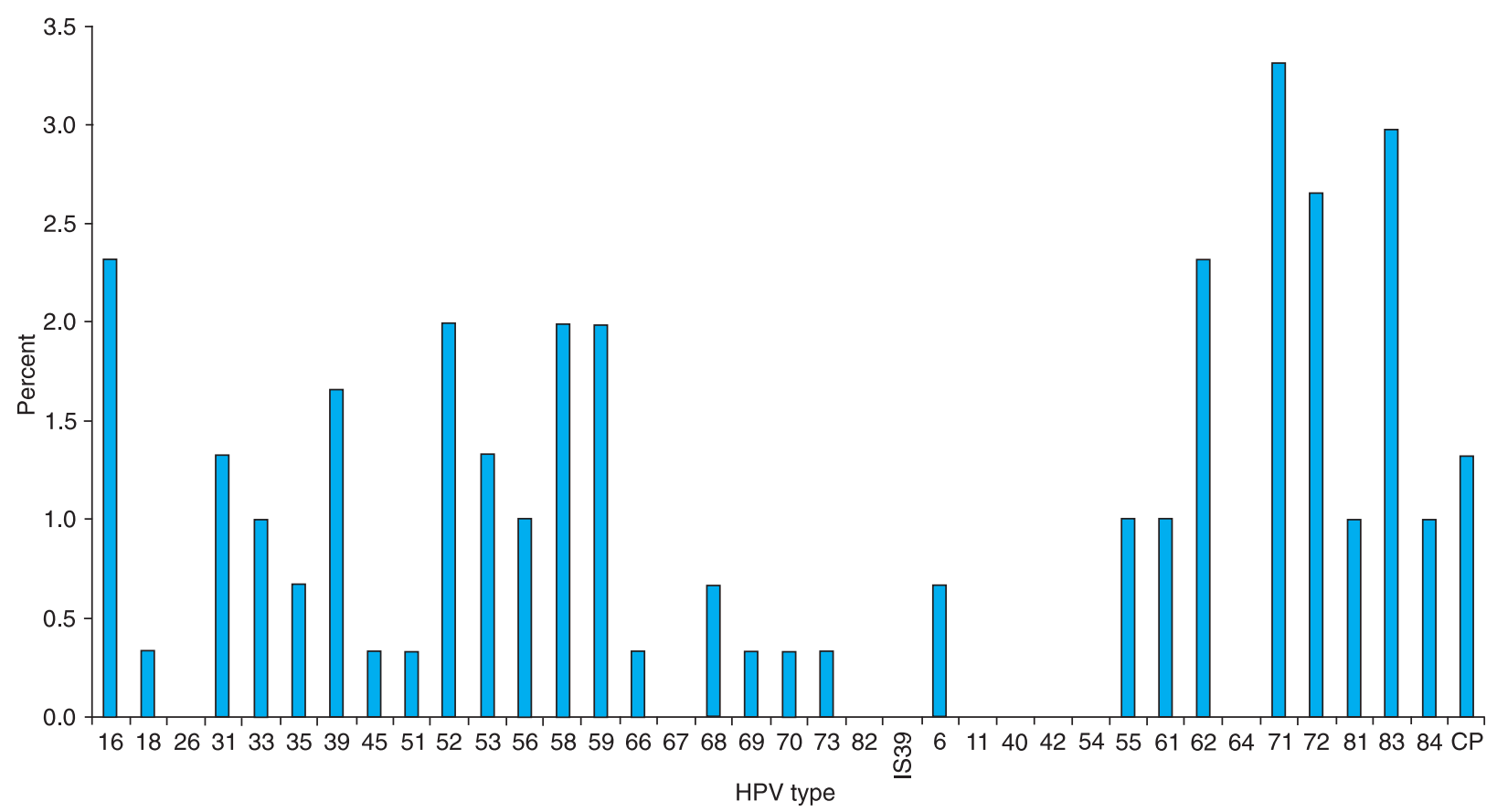

Figure 1. Detection of specific human papillomavirus (HPV) types in 302 participants. The percent of each HPV type detected is indicated on the $y$-axis and the specific HPV types that have been detected are indicated on the x-axis. A woman was considered to be infected with an HPV type if either the cervical or vaginal swab contained that HPV type.

swabs, 55 (18.2\%) were positive for any HPV, 36 swabs (11.9\%) were positive for 1 or more high-risk HPV types, and 43 vaginal swabs (14.2\%) were positive for 1 or more low-risk HPV types (Table 1).

There were many different HPV types detected in both types of swabs (Figure 2). The most frequently detected high-risk HPV types in cervical swabs were HPV types 16 (7 cases), 59 (5 cases), 31, 39, 58 (4 cases each), and 52 (3 cases). The most frequently detected low-risk HPV types in cervical swabs were HPV types 71 (8 cases) and 62 (4 cases).

The most frequently detected high-risk HPV types in vaginal swabs were HPV types 16 and 39 (6 cases each), 52 and 59 (5 cases each), and 53 and 58 (4 cases each). The most frequently detected low-risk HPV types in vagi- nal swabs were HPV types 71 and 83 (9 cases each), 72 (8 cases), and 62 (7 cases).

In general, there was a good correlation between the HPV types detected in the cervical and vaginal swabs from the participants. However, several types such as HPV types 72 and 83 were more frequently detected in vaginal swabs than in cervical swabs (Figure 2).

\section{Pap smear results and HPV detection}

Of all study participants, 285 (94.4\%) had normal Pap smears, and 17 (5.6\%) had abnormal Pap smears (Table 2). Of the 17 abnormal Pap smears, 13 were LGSIL and 4 were HGSIL. The diagnosis of atypical squamous cells of undetermined significance (ASCUS) was not made for any of the participants. This observation may have been a 


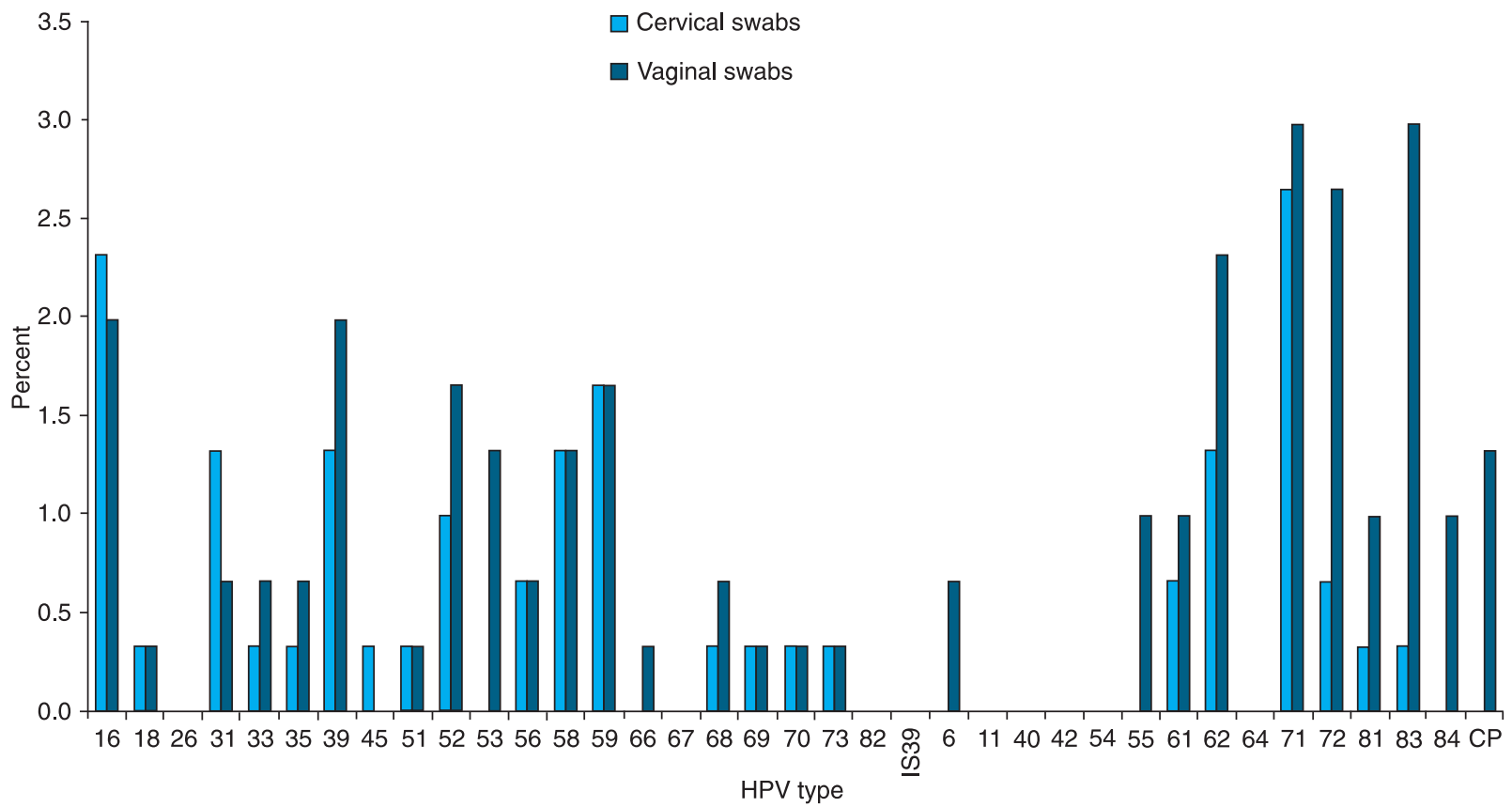

Figure 2. Detection of specific human papillomavirus (HPV) types in cervical and vaginal swab specimens from 302 women. The percent of each HPV detected in the swabs is indicated on the $y$-axis and the specific HPV types detected in the assay are shown on the $\mathrm{x}$-axis.

feature of local cytologic interpretation, since some of the LGSIL specimens (local interpretation) were interpreted as ASCUS by cytologists at Indiana University.

Of the 285 participants with normal Pap smears, 59 (20.6\%) were HPV-positive, and 31 (10.9\%) were high-risk HPV-positive. Of the 13 participants with Pap smears indicating LGSIL, 10 (76.9\%) were positive for HPV, and high-risk HPV types were detected in 9 (69.2\%) of these 13 women. All 4 participants with Pap smears indicating an HGSIL were positive for HPV in 1 or both swabs, and highrisk HPV types were detected in all 4 of these women.

Both HPV positivity $(P<0.0001)$ and high-risk HPV positivity $(P<0.0001)$ were associated with Pap smear abnormality. Lifetime number of sexual partners was strongly associated with abnormal Pap smear results $(P=$ 0.0064). Specifically, the odds of having an abnormal Pap smear increased by approximately $60 \%$ for each added sexual partner (odds ratio estimate $1.6 ; 95 \% \mathrm{Cl}=1.1-2.2$ ). These observations from Ecuador are consistent with studies of HPV detection and abnormal cervical cytology, and with previous studies conducted elsewhere that indicate

Table 2. Association of Pap smear results with human papillomavirus (HPV) detection.

\begin{tabular}{lccc}
\hline & Normal & LGSIL & HGSIL \\
\hline Number of participants & 285 & 13 & 4 \\
Any HPV-positive & $59(20.7 \%)$ & $10(76.9 \%)$ & $4(100 \%)$ \\
HR-HPV-positive & $31(10.9 \%)$ & $9(69.2 \%)$ & $4(100 \%)$
\end{tabular}

Data are reported as number with percent in parentheses. LGSIL = low-grade squamous intraepithelial lesions; HGSIL = highgrade squamous intraepithelial lesions; HR-HPV = high-risk or oncogenic HPV.

Table 3. Association of age with human papillomavirus (HPV) detection.

\begin{tabular}{lccccc}
\hline & 0 to 20 years & 21 to 30 years & 31 to 40 years & 41 to 50 years & 51 years and older \\
\hline Number of participants & 8 & 92 & 89 & 69 & 43 \\
Any HPV-positive & $3(37.5 \%)$ & $20(21.7 \%)$ & $20(22.5 \%)$ & $16(23.2 \%)$ & $12(27.9 \%)$ \\
HR-HPV-positive & $3(37.5 \%)$ & $9(9.8 \%)$ & $9(10.1 \%)$ & $12(17.4 \%)$ & $9(20.9 \%)$
\end{tabular}

Data are reported as number with percent in parentheses. Two participants did not provide age. HR-HPV = high-risk or oncogenic HPV. 
an increased chance of HPV infection in women with many lifetime sexual partners $(6,14)$.

As shown in Table 3, there was a modest increase in HPV and high-risk HPV detection in older women (51 years of age and older), consistent with previous studies of HPV epidemiology in Latin American women $(22,23)$. The number of pregnancies, a history of genital warts, smoking cigarettes, and marital status were not factors associated with HPV positivity, high-risk HPV positivity, or abnormal Pap smears.

\section{Discussion}

The aims of our study were to contribute to the knowledge of HPV prevalence and type distribution in a defined population of Ecuadorian women, and to evaluate the utility of vaginal swab specimens in these women. We sought to determine how often women attending a cervical cancer screening clinic in Santa Elena, Ecuador, had demonstrable HPV and abnormal cervical cytology. In addition, we examined several factors associated with the detection of HPV including age, number of lifetime sex partners, and Pap smear abnormalities. We found that the number of lifetime sexual partners was positively associated with detection of HPV and with abnormal pap smears.

Cervical cancer is caused by persistent infection with oncogenic HPV $(24,25)$. A better understanding of the natural history of HPV infection in women living in Ecuador is needed because cervical cancer is very common in this country. Many women in Ecuador do not undergo routine cervical cancer screening and cancer is often not detected until the late stages of disease. Routine cervical cancer screening is not always available to women in Ecuador and other countries due to economic and logistic obstacles (26).

The physicians who performed the survey, Pap smear, and HPV cervical and vaginal swabs were Ecuadorian gynecologists, and therefore the level of communication between the study participants and examiners was not compromised due to language or cultural barriers. Additionally, approximately $25 \%$ of the women enrolled in the study had never had a prior Pap smear. Having this first exam will potentially reduce the stigma of having gynecologic screening for these patients and the larger female community in the city. Also, although the Dacron swabs used for HPV testing were shipped over a large distance, adequate DNA was obtained from approximately $98 \%$ of the specimens, demonstrating that shipping did not greatly decrease the utility of the swabs.

Detection methods for HPV include hybrid capture and PCR (27). The hybrid capture assay uses pooled probes to detect HPV in specimens, provides no specific HPV type information, and cannot determine whether individual or multiple types are present. In addition, it is an expensive assay (approximately US $\$ 60$ per assay). PCR assays are used primarily in research involving HPV. PCR assays provide type-specific information, but are technically difficult and expensive.

This study has some limitations. Due to selection bias, the conclusions may not be applicable to the general population of Ecuadorian women. In particular, it is possible that our study underestimated the true prevalence of HPV infection in the community as a whole. The women who participated in this study were self-selected, as they presented themselves to the clinic for cancer screening or for gynecologic care. In order to self-present, therefore, a number of prerequisites had to have been met. Primarily, women had to know about the existence of the clinic (it opened approximately 9 months before the start of enrollment) and had to be aware of and be interested in gynecologic care. Nearly $75 \%$ of participants in our study had received a Pap smear previously. Thus, these women clearly had knowledge of the importance of Pap smears and also possessed the means to pay for the test (which was ultimately paid for by the study). It is possible that many women in the city of Santa Elena either did not know about the clinic's existence, the role of gynecologic care apart from obstetrical intervention during pregnancy and parturition, or that the clinic offered gynecologic cancer screening. Additionally, the women who did know about the clinic and were interested in gynecologic care must have had transportation to the clinic. The major mode of transportation within the city of Santa Elena is ambulation, and some "barrios" are 2 to $3 \mathrm{~km}$ from the clinic site. Therefore, we may have unintentionally excluded those women who were sicker or disabled in some way, and potentially therefore excluded women with symptomatic invasive cervical cancer. Finally, the major mode of information transmission in Santa Elena is by word-of-mouth, especially regarding health information. Therefore, it is possible that many women living in the outlying "barrios" did not know about the enrollment period of the study.

Women in Ecuador suffer from a high cervical cancer burden. Larger studies are needed to classify the high-risk HPV types that are most prevalent in the country, and to determine which HPV types are most associated with actual invasive cancer tissues. The present study demonstrated the expected association between high-risk HPV types and cervical dysplasia in this specific population. For improved prevention of HPV transmission and treatment of HPV-related lesions, access to screening and treatment must be improved. Ecuador ranks 110th of 174 listed 
countries for gross domestic product per capita, and many women have little to spend on their own health care (28). Many women do not go to a gynecologist unless symptoms such as vaginal bleeding are present, and often not unless these symptoms have been present for a long time, in order to avoid incurring a health-care cost.

How can the burden of cervical dysplasia and cancer be reduced in Ecuador and other Latin American countries with limited resources? Behavioral measures including delaying sexual intercourse, the regular use of latex condoms, which has been shown to reduce the transmission of HPV $(29,30)$, and limiting the number of lifetime sexual partners may all be of benefit. Because of the barriers to Pap smear screening and treatment of precan- cerous lesions, the HPV vaccine might be an ideal way to protect women in Ecuador from invasive cervical cancer. Currently, the cost of the vaccine is all but prohibitive for these women, who are at high risk due to their reduced access to screening. Therefore, we urge the manufacturers of the newly approved HPV vaccines to provide vaccine free of charge or at a reduced cost to the women of Ecuador, thus making this mode of prevention accessible to those women who most need it. Our data may serve to encourage new and larger studies to determine the prevalence of HPV in the general population of Ecuadorian women who do not necessarily have access to cervical cancer screening.

\section{References}

1. Hildesheim A, Han CL, Brinton LA, Kurman RJ, Schiller JT. Human papillomavirus type 16 and risk of preinvasive and invasive vulvar cancer: results from a seroepidemiological case-control study. Obstet Gynecol 1997; 90: 748-754.

2. Future Directions in Epidemiologic and Preventive Research on Human Papillomaviruses and Cancer. Proceedings of a workshop. Bethesda, Maryland, USA, June 2002. J Natl Cancer Inst Monogr 2003; 1-130.

3. Dillner J, von Krogh G, Horenblas S, Meijer CJ. Etiology of squamous cell carcinoma of the penis. Scand J Urol Nephrol Suppl 2000; 205: 189-193.

4. zur Hausen $\mathrm{H}$. Viruses in human cancers. Eur $\mathrm{J}$ Cancer 1999; 35: 1174-1181.

5. zur Hausen H. Papillomaviruses and cancer: from basic studies to clinical application. Nat Rev Cancer 2002; 2: 342350.

6. Bosch FX, de Sanjose S. The epidemiology of human papillomavirus infection and cervical cancer. Dis Markers 2007; 23: 213-227.

7. Herrero R, Munoz N. Human papillomavirus and cancer. Cancer Surv 1999; 33: 75-98.

8. Gissmann L, Wolnik L, Ikenberg H, Koldovsky U, Schnurch HG, zur Hausen H. Human papillomavirus types 6 and 11 DNA sequences in genital and laryngeal papillomas and in some cervical cancers. Proc Natl Acad Sci U S A 1983; 80: 560-563.

9. Pfister H. Human papillomaviruses and genital cancer. Adv Cancer Res 1987; 48: 113-147.

10. Brown DR, Schroeder JM, Bryan JT, Stoler MH, Fife KH. Detection of multiple human papillomavirus types in condylomata acuminata lesions from otherwise healthy and immunosuppressed patients. J Clin Microbiol 1999; 37: 33163322.

11. Parkin DM, Bray F, Ferlay J, Pisani P. Global cancer statistics, 2002. CA Cancer J Clin 2005; 55: 74-108.

12. Parkin DM, Pisani P, Ferlay J. Estimates of the worldwide incidence of eighteen major cancers in 1985. Int $J$ Cancer 1993; 54: 594-606.
13. Pisani P, Parkin DM, Munoz N, Ferlay J. Cancer and infection: estimates of the attributable fraction in 1990. Cancer Epidemiol Biomarkers Prev 1997; 6: 387-400.

14. Schiffman M, Castle PE, Jeronimo J, Rodriguez AC, Wacholder S. Human papillomavirus and cervical cancer. Lancet 2007; 370: 890-907.

15. Arrossi S, Sankaranarayanan R, Parkin DM. Incidence and mortality of cervical cancer in Latin America. Salud Publica Mex 2003; 45 (Suppl 3): S306-S314.

16. Bosetti C, Malvezzi M, Chatenoud L, Negri E, Levi F, La Vecchia C. Trends in cancer mortality in the Americas, 1970-2000. Ann Oncol 2005; 16: 489-511.

17. Robles SC, White F, Peruga A. Trends in cervical cancer mortality in the Americas. Bull Pan Am Health Organ 1996; 30: 290-301.

18. Paez C, Konno R, Yaegashi N, Matsunaga G, Araujo I, Corral F, et al. Prevalence of HPV DNA in cervical lesions in patients from Ecuador and Japan. Tohoku J Exp Med 1996; 180: 261-272.

19. Hurtig AK, San Sebastian M. Gynecologic and breast malignancies in the Amazon basin of Ecuador, 1985-1998. Int J Gynaecol Obstet 2002; 76: 199-201.

20. Gravitt PE, Peyton CL, Alessi TQ, Wheeler CM, Coutlee F, Hildesheim A, et al. Improved amplification of genital human papillomaviruses. J Clin Microbiol 2000; 38: 357-361.

21. Brown DR, Legge D, Qadadri B. Distribution of human papillomavirus types in cervicovaginal washings from women evaluated in a sexually transmitted diseases clinic. Sex Transm Dis 2002; 29: 763-768.

22. Herrero R, Castle PE, Schiffman M, Bratti MC, Hildesheim A, Morales $\mathrm{J}$, et al. Epidemiologic profile of type-specific human papillomavirus infection and cervical neoplasia in Guanacaste, Costa Rica. J Infect Dis 2005; 191: 17961807.

23. Castle PE, Schiffman M, Herrero R, Hildesheim A, Rodriguez $A C$, Bratti MC, et al. A prospective study of age trends in cervical human papillomavirus acquisition and persistence in Guanacaste, Costa Rica. J Infect Dis 2005; 191: 
1808-1816.

24. zur Hausen H. Papillomaviruses in human cancers. Proc Assoc Am Physicians 1999; 111: 581-587.

25. Munoz N. Human papillomavirus and cancer: the epidemiological evidence. J Clin Virol 2000; 19: 1-5.

26. Agurto I, Bishop A, Sanchez G, Betancourt Z, Robles S. Perceived barriers and benefits to cervical cancer screening in Latin America. Prev Med 2004; 39: 91-98.

27. van Doorn LJ, Kleter B, Quint WG. Molecular detection and genotyping of human papillomavirus. Expert Rev Mol Diagn 2001; 1: 394-402.
28. Program UND. Human development reports: Ecuador. http:// hdrstats.undp.org/countries/country_fact_sheetscty_fs_ ECU.html.

29. Bleeker MC, Berkhof J, Hogewoning CJ, Voorhorst FJ, van den Brule AJ, Starink TM, et al. HPV type concordance in sexual couples determines the effect of condoms on regression of flat penile lesions. Br J Cancer 2005; 92: 1388-1392.

30. Holmes KK, Levine R, Weaver M. Effectiveness of condoms in preventing sexually transmitted infections. Bull World Health Organ 2004; 82: 454-461. 